\title{
Intractable Hiccups and Gastroesophageal Reflux Disease Attributable to Brain Tumor: A Case Report
}

\author{
Voranush Chongsrisawat $^{1 *}$, Kantida Ongasivachai ${ }^{1}$, Chidchanok Tangjade ${ }^{1}$, Netsiri Dumrongpisutikul ${ }^{2}$ \\ and Jiraphorn Amornfa ${ }^{3}$
}

${ }^{1}$ Department of Pediatrics, Chulalongkorn University, Thailand

${ }^{2}$ Department of Radiology, Chulalongkorn University, Thailand

${ }^{3}$ Department of Surgery, Chulalongkorn University, Thailand

Received: January 27, 2018; Published: February 06, 2018

*Corresponding author: Voranush Chongsrisawat, Department of Pediatrics, Faculty of Medicine, Chulalongkorn University, Bangkok 10330, Thailand, Email: voranush.c@chula.ac.th

\begin{abstract}
We report a 6-year-old girl who presented with intractable hiccups and epigastria pain. Gastroesophageal reflux disease (GERD) was confirmed by esophageal pH monitoring and EGD revealed an esophageal ulcer and erosion at distal esophagus. Treatment with high dose PPI failed to reduce hiccups. MRI of brain demonstrated enhancing infiltrative lesion involved floor of forth ventricle, posterior aspect of lower pons, entire medulla, cervicomedullary junction and extended down to dorsal portion of C7 cord. This report emphasizes brain tumor as the etiology of intractable hiccups leading to severe GERD. When encountering a patient with intractable hiccups, appropriate CNS imaging studies should be included in the evaluation protocol.
\end{abstract}

Keywords: Intractable hiccups; Gastroesophageal reflux disease; Esophagitis; Esophageal ulcer; Brainstem tumor

Abbreviations: GERD: Gastroesophageal Reflux Disease; CNS: Central Nervous System

\section{Introduction}

Hiccup or singultus is a reflex inspiratory movement consisting of involuntary, repeated, spasmodic contraction of the diaphragm and intercostal muscles followed by abrupt closure of the glottis [1]. Hiccup is usually a self-limited disorder without any clinical significance [2], most commonly caused by gastric distension (ie. food, air), alcohol ingestion, tobacco use, or sudden changes of gastrointestinal temperatures [2,3]. Episodes lasting longer than 48 hours are defined as persistent, and those lasting longer than two months are labeled as intractable [1]. Intractable hiccups have various etiologies including vagus or phrenic nerve irritation, toxicmetabolic disorders, central nervous system (CNS) disorders, and psychogenic causes. We reported a child with intractable hiccups and severe Gastroesophageal reflux disease resulting from probable infiltrating astrocytoma at brainstem and cervical spinal cord.

\section{Case Report}

A 6-year-old girl presented with hiccups, snoring, and anemia for 2 months. Hiccups persisted throughout day and night. She also had epigastric pain, nausea, and loss of appetite. Her BW was $19.6 \mathrm{~kg}\left(50^{\text {th }}\right.$ percentile) and height was $109 \mathrm{~cm}\left(25^{\text {th }}\right.$ percentile). Her general medical examination revealed pallor and unremarkable otherwise. There was no papilledema. Motor, sensory, and cerebellar function were intact. Her hemoglobin was $8.6 \mathrm{~g} / \mathrm{dL}$, with normal leukocyte and platelet count. Gastroesophageal reflux disease was assumed as the cause of her symptoms, therefore she was put on $20 \mathrm{mg}$ of omeprazole daily. One month later, she had hematemesis. EGD revealed an esophageal ulcer and erosion at distal esophagus (Figure 1). Her symptoms continued even she was on $60 \mathrm{mg}$ of lansoprazole and iron supplement. Esophageal $\mathrm{pH}$ monitoring revealed a reflux index of 59\% and impedance study demonstrated 65 episodes of acid reflux.

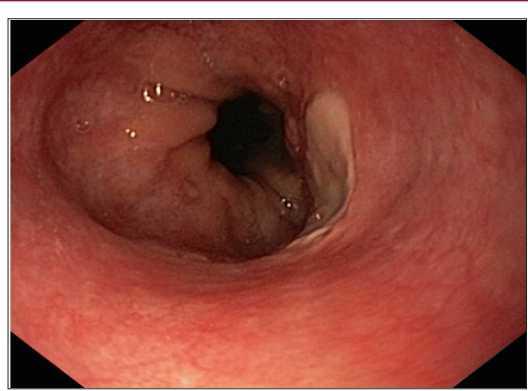

Figure 1: EGD revealed an oesophageal ulcer and erosion at distal oesophagus. 
Upper GI series was unremarkable, Because of unremitting hiccups, brain imaging was conducted, and magnetic resonance imaging demonstrated enhancing infiltrative lesion involved floor of forth ventricle, posterior aspect of lower pons, entire medulla, cervicomedullary junction and extended down to dorsal portion of
C7 cord (Figure 2). Biopsy revealed reactive gliosis. Her symptoms improved with radiotherapy. Subsequently she underwent complete tumor resection which revealed desmoid tumor. After 5 years, the patient is in good clinical condition without hiccup.
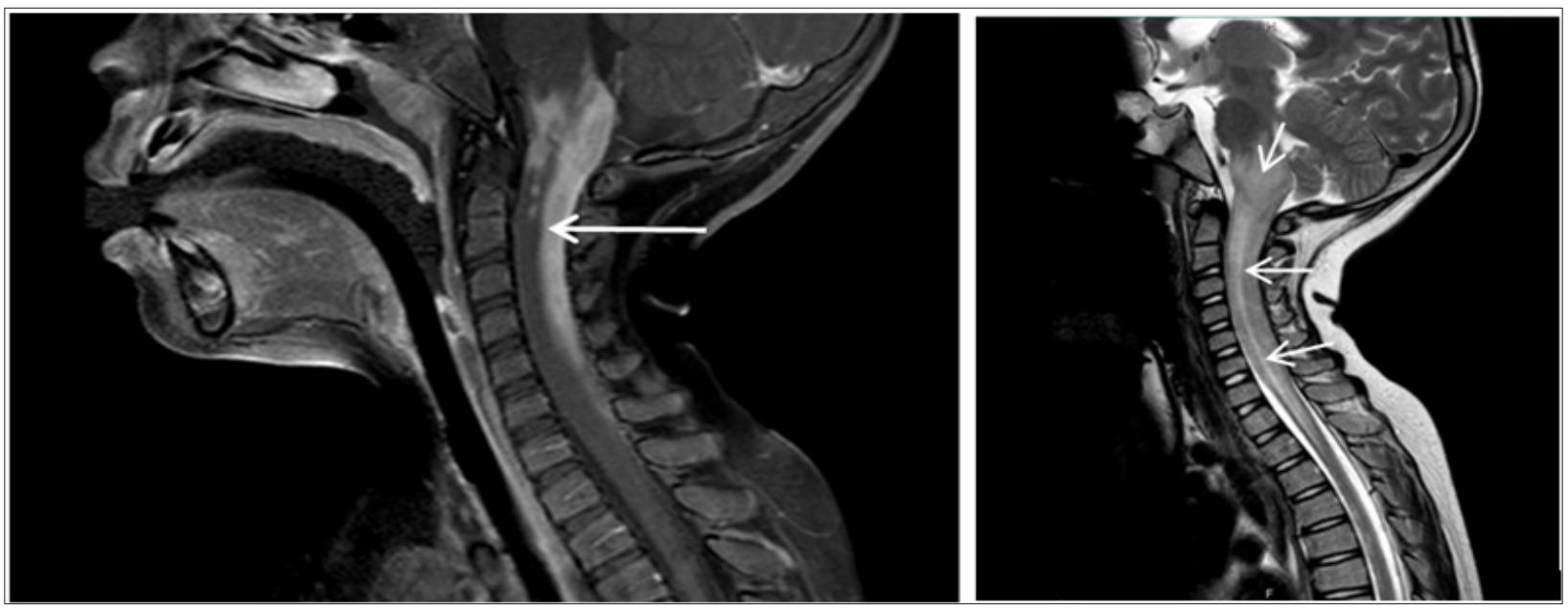

Figure 2: MRI showed enhancing infiltrative lesion involved floor of 4th ventricle, posterior aspect of lower pons, entire medulla, cervicomedullary junction and extended down to dorsal portion of C7 cord without leptomeningeal seeding (A: Gdenhanced T1WI, B: T2WI).

\section{Discussion}

Intractable hiccups can be caused by a disease or lesion along the hiccups reflex arc [4]. Gastrointestinal disease is the most frequent cause; about two thirds of these patients have a Gastroesophageal reflux [3,5]. Atypical or extraesophageal presentations of GERD are increasingly documented and consist mainly of pulmonary and otolaryngologic symptoms. Yi, et al. reported hiccup symptoms in 55\% of patients with GERD [6]. The mechanisms have been postulated as to how reflux occurs during hiccups is negative intra esophageal pressure and a reduction in LES pressure during hiccups result in movement of acid from the stomach into the esophagus resulting in esophagitis. Another mechanism is the repetitive diaphragmatic contractions that occur with hiccups resulting in increased gastric pressure, thus facilitating acid reflux. Complications of reflux such as strictures, esophagitis, and esophageal ulcer may stimulate vagal nerves that may result in intractable hiccups [7].

Intractable hiccups may result from serious pathological processes due to central nervous system disorders mostly caused by brainstem lesions such as trauma, ischemic stroke, infection, neuromyelitis optical and particularly brainstem tumor $[1,3,4,8]$. Hiccups have been implicated as a cause of severe reflux esophagitis $[7,9]$. However there has been no report of esophageal ulcer caused by intractable hiccups as our case. Therapy with potent PPI can differentiate hiccups caused by esophagitis and hiccups-induced esophagitis $[3,5,10]$. Brain MRI is indicated in patients whose hiccups fail to respond to high dose PPI as in our case.

Brain tumor presenting with hiccups is a rare condition in children. There have been a few case reports of brain tumor causing intractable hiccups in children. All reported patients had abnormality of neurological examination such as brisk deep tendon reflex, tongue fasciculation, and horizontal nystagmus [11,12]. This reported case had no neurological signs and symptoms and thus underwent multiple investigations for GERD. This report emphasizes brain tumor as the etiology of intractable hiccups. When encountering a patient with intractable hiccups even if the patients do not reveal any neurological manifestations, appropriate CNS imaging studies should be included in the evaluation protocol.

\section{References}

1. Becker DE (2010) Nausea, vomiting, and hiccups: a review of mechanisms and treatment. Anesth Prog 57: 150-157.

2. Chang FY, Lu CL (2012) Hiccup: mystery, nature and treatment. J Neurogastroenterol Motil 18: 123-130.

3. Marsot-Dupuch K, Bousson V, Cabane J, Tubiana JM (1995) Intractable hiccups: the role of cerebral MR in cases without systemic cause. AJNR Am J Neuroradiol 16: 2093-2100.

4. Arami MA (2010) A case of brainstem cavernous angioma presenting with persistent hiccups. Acta Med Iran 48: 277-278.

5. Launois S, Bizec JL, Whitelaw WA, Cabane J, Derenne JP (1993) Hiccup in adults: an overview. Eur Respir J 6: 563-575.

6. Yi CH, Liu TT, Chen CL (2012) A typical symptoms in patients with gastroesophageal reflux disease. J Neurogastroenterol Motil 18: 278283.

7. Pooran N, Lee D, Sideridis K (2006) Protracted hiccups due to severe erosive esophagitis: a case series. J Clin Gastroenterol 40: 183-185.

8. McKeon A, Lennon VA, Lotze T, Tenenbaum S, Ness JM, et al. (2008) CNS aquaporin-4 autoimmunity in children. Neurology 71: 93-100.

9. Lewis JH (1985) Hiccups: causes and cures. J Clin Gastroenterol 7: 539552. 
10. Shay SS, Myers RL, Johnson LF (1984) Hiccups associated with reflux esophagitis. Gastroenterology 87: 204-207.

11. Tanaka Y, Koga Y, Takada H (2015) Pilocytic astrocytoma at the medulla oblongata dorsal surface presenting as intractable hiccups. Pediatr Neurol 52: 254-255.

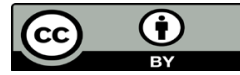

This work is licensed under Creative Commons Attribution 4.0 License

Submission Link: http://biomedres.us/submit-manuscript.php
12. Fischer AQ McLean WT (1982) Intractable hiccups as presenting symptom of brainstem tumor in children. Childs Brain 9: 60-63.

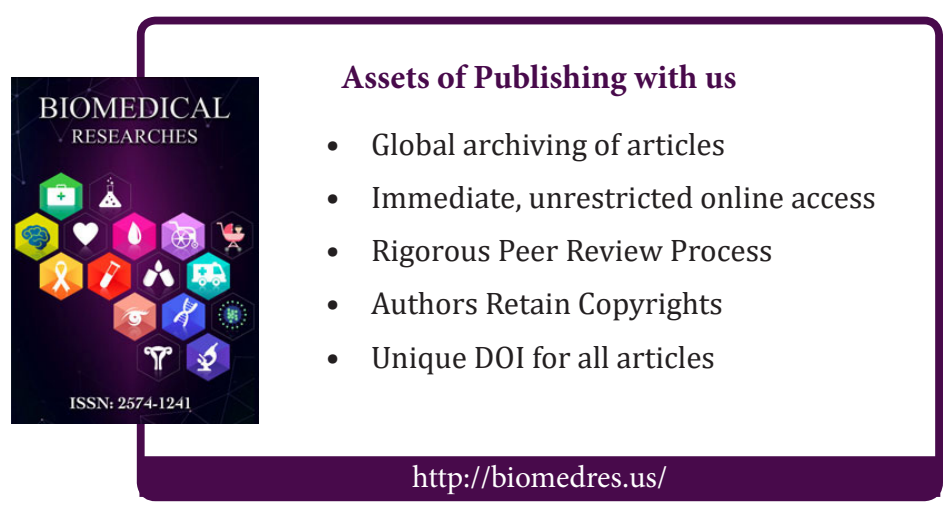

